\title{
The Great Migration: How MRI Replaces Traditional Imaging Techniques for the Characterization of Atherosclerosis
}

\author{
T. C. Walter · G. Knobloch • M. Kasner • \\ G. Diederichs · B. Hamm • U. Ebersberger • \\ M. R. Makowski
}

Published online: 14 February 2014

(C) Springer Science+Business Media New York 2014

\begin{abstract}
In the Western world and developing countries, cardiovascular diseases remain the primary cause of morbidity and mortality. The incidence of cardiovascular diseases is thought to be on the rise, due to an increase in risk factors (e.g. diabetes) and aging of the population. Currently, there is no established clinical method for the in vivo characterization of atherosclerotic plaques and the prediction of ischemic stroke, myocardial infarction and other clinical complications. In this article, we will introduce novel magnetic resonance imaging (MRI) techniques and discuss how these techniques are starting to replace currently established clinical imaging methods for the detection and characterization of atherosclerotic vessel wall changes.
\end{abstract}

T. C. Walter and G. Knobloch have contributed equally.

This article is part of the Topical Collection on New Imaging Technologies.

T. C. Walter · G. Knobloch · G. Diederichs · B. Hamm •

M. R. Makowski ( $\bowtie)$

Department of Radiology, Charité Universitätsmedizin, Berlin, Germany

e-mail: marcusmakowski@gmail.com

M. Kasner

Department of Cardiology, Charité Universitätsmedizin, Berlin, Germany

U. Ebersberger

Department of Cardiology and Intensive Care Medicine, Heart Center Munich-Bogenhausen, Munich, Germany

M. R. Makowski

Division of Imaging Sciences, King's College London, London, UK
Keywords MRI $\cdot$ Molecular imaging $\cdot$ Cardiovascular disease $\cdot$ Atherosclerosis

\section{Introduction}

In the Western world and developing countries, cardiovascular diseases remain the primary cause of overall morbidity and mortality in the general population [1]. The incidence of cardiovascular diseases, including myocardial infarction, cerebral stroke and abdominal aortic aneurysms, are thought to increase in the future, due to a considerable projected increase in risk factors (e.g. diabetes, obesity) in an aging population. Currently, there are no clinically established methods available for the in vivo characterization of atherosclerotic plaques or the prediction of plaque rupture, which in most cases are the cause of myocardial infarction, ischemic stroke and other severe complications. In most cases, the role of the currently established cardiovascular imaging modalities is limited to the assessment of the degree of luminal stenosis, or the effect this stenosis has on myocardial or cerebral blood flow and perfusion. The degree of stenosis is currently regarded as the gold standard for the clinical evaluation of carotid and coronary artery disease, and treatment decisions are made on the basis of these luminographic measurements. The main limitation of the parameter "degree of stenosis" is that it does not allow the differentiation of stable and unstable atherosclerotic plaques [2••]. Therefore, most cardiovascular events cannot be predicted based on this parameter.

Stable atherosclerotic plaques tend to develop gradually over time, sometimes decades, and do not have a tendency for sudden rupture. Often, this type of atherosclerotic plaque can be detected and treated before the associated 
plaque rupture results in cardiovascular events. For this type of atherosclerotic plaque the degree of stenosis is a relevant and clinically useful parameter. On the other hand, unstable atherosclerotic plaques bear the risk of sudden rupture. Plaque rupture plays a major contribution to the high mortality and morbidity associated with atherosclerotic vessel wall diseases. The detection of these unstable or vulnerable atherosclerotic plaques remains challenging. An increasing number of studies has demonstrated that the majority of unstable or ruptured atherosclerotic plaques can be found in arteries with a degree of stenosis of less than $60 \%$ [3-5]. This is an alarming observation and explains why this type of atherosclerotic plaque cannot be detected with the currently used imaging techniques. Therefore, new imaging methods and techniques are urgently needed to enable the early detection and characterization of these unstable atherosclerotic plaques. Various previous studies have indicated that noninvasive characterization of plaque composition and visualization of the biological processes on a molecular level are promising approaches for early detection of unstable atherosclerotic plaques.

Currently, clinically established imaging methods rely on the quantification of the degree of stenosis, which is still the reference standard in clinical evaluation of carotid and coronary artery disease. Most treatment decisions are based on these luminographic measurements. A good example, which illustrates the limitations of these methods, is a process referred to as "positive vascular remodeling", which occurs during plaque formation. Atherosclerotic plaques, especially unstable atherosclerotic plaques, progress not only towards the vascular lumen (negative remodeling), but also grow away from the vascular lumen (positive remodeling). In the latter case, plaques will not cause significant narrowing of the artery, despite a significant overall enlargement of the plaque. This progression pattern is a typical feature of unstable atherosclerotic plaques [6], and explains why this type of plaque cannot be detected by luminographic imaging methods or techniques for the quantification of myocardial or cerebral perfusion of blood flow [7].

Compared to these imaging modalities, non-contrast enhanced and contrast enhanced magnetic resonance imaging (MRI) is an excellent imaging tool for the characterization of atherosclerotic plaques. Today, non-contrast enhanced MRI is feasible in the visualization and characterization of different atherosclerotic plaque components in the coronary artery, carotid artery and aorta. Clinically evaluated unspecific MRI contrast agents also enable a sensitive and more detailed visualization of other plaque components, e.g. the fibrous cap and necrotic core. The use of specifically targeted molecular MR probes permit detection and quantification of specific molecules, proteins or cells within the plaque. Such probes could enable an early and specific detection of molecular processes during disease development, and thereby facilitate the early detection of unstable atherosclerotic plaques. Detailed detection and imaging of this plaque formation process could represent a novel diagnostic basis upon which treatment decisions can made, and response to therapy could be measured and quantified.

In this article, we will introduce novel MRI techniques, and discuss how these techniques are beginning to replace currently established clinical cardiovascular imaging methods for the detection and characterization of atherosclerotic vessel wall changes.

\section{Biological Background of Atherosclerotic Vessel Wall Changes}

Atherosclerosis is slow-progressing disease (Fig. 1). The earliest changes, which lead to the development of atherosclerotic plaques, occur in the arterial intima of the affected artery. If the endothelial barrier is damaged by for example toxins, it becomes leaky and an influx of cholesterol and modified lipids cannot be prevented anymore [8•]. This represents the initial event and a starting point from which atherosclerotic plaques begin to develop. Low-density lipoproteins (LDL) are considered to be precursors of atherosclerotic plaques. The accumulation of LDL in early plaque-stages leads to a local inflammatory process, which subsequently results in the activation of endothelial cells. After activation, these cells express certain signaling molecules on their cellular surface, e.g. vascular-celladhesion-molecule-1 (VCAM-1). These surface markers then enable and catalyze the recruitment and influx of more inflammatory cells from the blood stream into atherosclerotic plaques. Within the atherosclerotic plaque, inflammatory cells start to phagocytose modified LDL particles. During the progression of atherosclerosis, activated macrophages accumulate cholesterol, one of the main molecules within the plaque. Subsequently macrophages transform into so-called foam cells. During this process, macrophages also express proinflammatory markers, e.g. different types of matrix metalloproteinases and cytokines. These markers attract more proinflammatory cells that contribute to a further destabilization of the atherosclerotic plaque [8•]. Apart from macrophages and leukocytes, smooth muscle cells play an important role in the initiation and progression of atherosclerotic plaques. Smooth muscle cells are mainly responsible for the expression of extracellular matrix proteins, such as collagen and elastin. Extracellular matrix proteins represent a major component of atherosclerotic plaques in the different phases of plaque progression. The relative composition of the atherosclerotic plaque regarding different plaque components, e.g. the necrotic core and extracellular matrix, has been shown to 


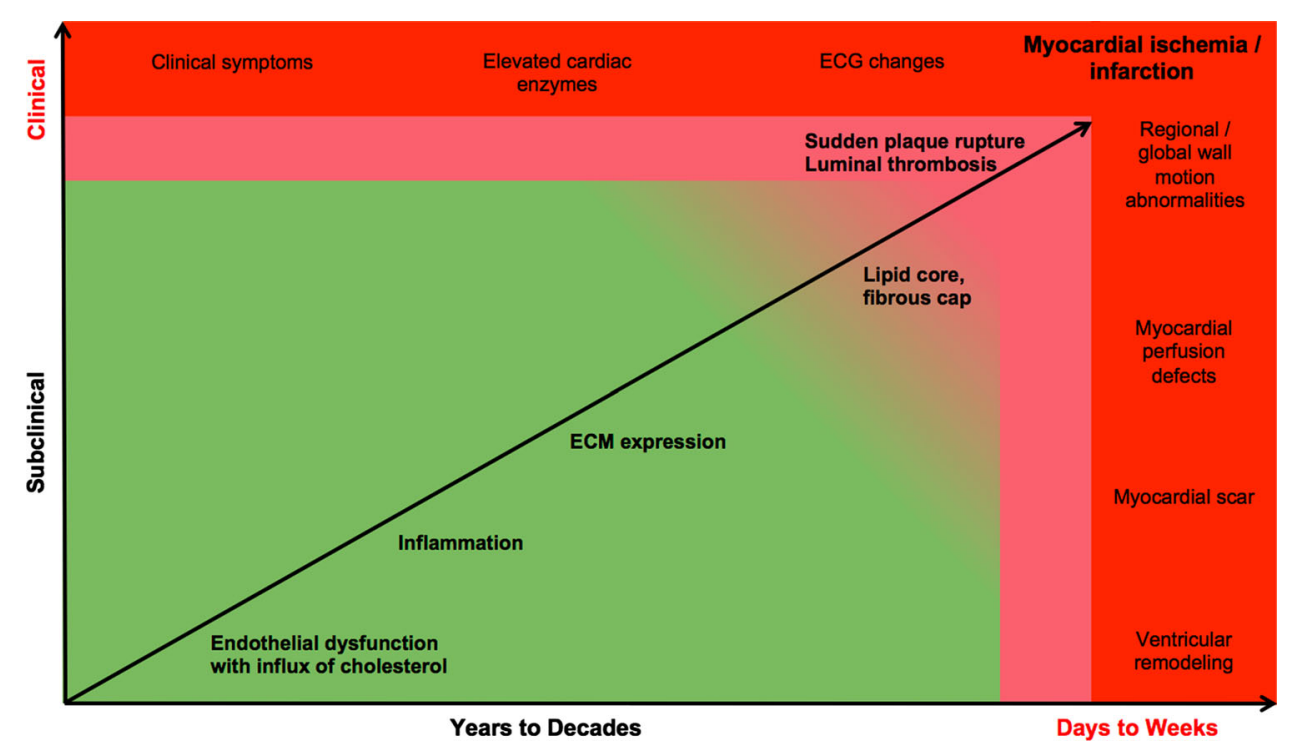

Fig. 1 Time course of the development of atherosclerotic lesions. Atherosclerosis is a slowly developing disease and goes through different phases. One of the earliest processes that can be observed is the influx of cholesterol into the arterial intima. Hence, a local inflammatory process is initiated, with the subsequent expression of extracellular matrix proteins and the formation of a lipid core including a fibrous cap. In contrast to these very slowly developing processes, plaque rupture with associated luminal thrombosis occurs within days. Consequences of sudden plaque rupture can be myocardial infarction and ischemic stroke allow a differentiation of atherosclerotic plaques regarding their stability. If a large necrotic core develops, which mainly consists of modified lipids and cholesterol cells, atherosclerotic plaques are considered to be unstable or vulnerable [9]. If the extracellular matrix represents the major part of the matrix, with elastin and collagen as main components, atherosclerotic plaques are considered as stable [2••]. These classifications are derived from various in vivo (e.g. intravascular ultrasound) and ex vivo (e.g. postmortem) studies, which have extensively characterized ruptured and un-ruptured atherosclerotic plaques in a large number of patients $[2 \bullet \bullet, 8 \bullet]$.

Other properties, which are associated with plaque instability, include neoangiogenesis (new vessel formation) within the plaque. Especially in advanced atherosclerotic plaques, these new vessels can become leaky and cause an accumulation of blood in the plaque matrix or intraplaque hemorrhage.

Apart from these differences in plaque composition, it has also been shown that the overall size of the plaque, the atherosclerotic plaque burden, is an indicator for plaque instability. It is, however, important to mention that plaque burden cannot be assessed with luminographic methods, e.g. catheter angiography. This is owing to the fact that atherosclerotic plaques expand not only into the direction of the vascular lumen (negative remodeling), but also towards the vessel wall (positive remodeling). Hence, luminographic methods can only detect the degree of the negative remodeling. Various studies have shown that the degree of luminal stenosis is not a parameter that allows noninvasive differentiation of stable and unstable atherosclerotic plaques [2•]. This is of high significance, as current treatment decisions are nonetheless based on the measurement of the degree of luminal stenosis.

The coronary arteries and the carotid arteries are the two main vascular beds in which unstable atherosclerotic plaques develop. In consequence, these two vascular beds have been extensively investigated and characterized in the context of atherosclerosis [10]. Many overlapping features exist between the properties and the composition of carotid and coronary atherosclerotic plaques, but there are also some differences. It is thought that most of these differences are the result of a higher arterial flow in the carotid arteries compared to the coronary arteries. The higher arterial flow causes stronger shear forces to act on the vessel wall, which affects the endothelial surface of the atherosclerotic plaque. Therefore, a higher percentage of plaque ulcerations are observed in the carotid arteries [11]. Even though there are some distinct differences, it was shown for both the coronary and carotid arteries that rupture of unstable atherosclerotic lesions leads to symptomatic disease, and that the degree of luminal stenosis does not allow a distinction between unstable and stable plaques. Currently, as already described for the coronary arteries, treatment decisions regarding the management of carotid artery disease are also based on the degree of luminal stenosis. 


\section{Principles of Molecular Magnetic Resonance Imaging}

In molecular magnetic resonance imaging, specifically targeted molecular probes are used for the detection and quantification of certain proteins, molecules or cell types in vivo. Molecular magnetic resonance imaging is an emerging technology compared to already established nuclear molecular imaging techniques, which include single photon emission computed tomography (SPECT) and positron emission tomography (PET). The main advantage of molecular MRI, especially in the context of atherosclerosis, is that imaging can be performed with a high spatial resolution. This allows not only the detection of the molecular probe, but also the characterization of the spatial distribution of the probe within the plaque. This unique information cannot be derived from nuclear imaging methods, as these techniques provide a low spatial resolution compared to MRI. The advantage of nuclear imaging techniques, however, is that the sensitivity for the detection of the molecular probe is higher [12]. A major disadvantage of nuclear imaging techniques is the need for ionizing radiation.

There are various types of molecular MR agents [13]. Iron-oxide-based and gadolinium-based molecular MR probes are most commonly used. Depending on the type of contrast agent, they affect either the longitudinal (T1) relaxation time or the transverse (T2) relaxation time of water protons. These effects can be detected with specific MR sequences. This kind of contrast mechanism is the main difference between MR and nuclear probes. Nuclear probes are detected directly, due to the radiation they emit. MR contrast agents, on the other hand, can only be detected indirectly by visualizing the effect they have on surrounding water protons.

The design of a molecular MRI probe determines whether the molecular probe binds actively or passively to its molecular target. Actively binding molecular probes selectively bind to specific molecular targets. In most cases, the binding mechanism is based on specific peptides or antibodies on the surface of the probe, which have a high affinity for a specific molecule or protein. Passively binding molecular probes are indirectly bound to their molecular target. Iron oxide based particles are an example of a particle with this type of binding mechanism; the iron oxide particles are phagocytosed by activated macrophages and are therefore specifically accumulated in this type of proinflammatory cell. Macrophages incorporate the particles and label themselves with the molecular probe. And thus, they become detectable by MRI.

Iron-oxide-based molecular probes belong to the group of $\mathrm{T} 2$ and $\mathrm{T} 2 *$ shortening agents. The effect this type of molecular probe has on the $\mathrm{T} 2$ and $\mathrm{T} 2 *$ relaxation time is much stronger compared to the effect it has on the T1 relaxation time $[14,15]$. The shortening of the $\mathrm{T} 2$ and $\mathrm{T} 2 *$ relaxation times leads to signal voids in the acquired image, by which these molecular probes can be detected [16]. Iron-oxide-based molecular probes have been extensively tested and evaluated in preclinical and clinical MRI studies in the context of atherosclerosis. In recent decades, various research groups and companies have put effort in the development and optimization of these particles. A variety of different forms and compositions of iron oxides particles have been developed and tested for different applications [17]. Iron-oxide-based molecular probes can be categorized into groups with a long and a short blood half-life. Superparamagnetic iron oxide particles (SPIO) are particles with a short blood half-life. The main advantage of these particles is that they are rapidly cleared from the blood stream by the liver and the reticuloendothelial system. Due to the fact that these particles specifically accumulate in these tissues, they have been studied extensively for imaging and characterization of the liver and of lymph nodes.

Ultrasmall superparamagnetic iron oxide particles (USPIO) are particles with a long blood half-life. As the particles are typically coated by for example different types of dextrans, they do not tend to aggregate and remain in a monodispersed form in solution. Due to this difference in composition, they remain in the blood stream much longer. The long circulation time-in some cases more than $24 \mathrm{~h}$ [17]-allows macrophages in the blood stream to phagocytose the particles. These iron-oxide-labeled macrophages ultimately migrate to areas of focal inflammation, where they can be detected and quantified by molecular MRI. As a result of the specific properties of these long circulating particles, they have mainly been used to study inflammatory processes, including the inflammatory burden of atherosclerotic plaques. As iron oxide particles mainly shorten $\mathrm{T} 2$ and $\mathrm{T} 2 *$ relaxation times, they are usually visualized with strongly weighted $\mathrm{T} 2$ or $\mathrm{T} 2 *$ sequences [18].

Gadolinium-based molecular probes belong to the group of T1 shortening agents. These types of molecular agents have a more pronounced effect on the $\mathrm{T} 1$ relaxation time compared to the $\mathrm{T} 2$ relaxation time. Therefore, they can be detected as an increase of signal, i.e. positive signal on T1 weighted images. The shortening of the $\mathrm{T} 1$ relaxivity by this type of molecular probe depends on different factors and properties, including the hydration number, the rotational correlation frequency, as well as the exchange rate of surrounding water protons [15]. Molecular T1 MR probes are usually designed to be target-specific by conjugating the signaling part (e.g. a gadolinium chelate) of the agent to a specific ligand, e.g. affibodies, peptides, binding parts of antibodies or small molecules. The binding of the molecular probe to its target significantly reduces the molecular 
rotation frequency of the probe, which increases the interaction of the gadolinium chelate with surrounding water molecules. This process leads to an increase of the relaxivity (R1) of the probe, and is referred to as receptorinduced magnetization enhancement (RIME). This explains why bound molecular MRI probes have a higher T1 relaxivity compared to unbound probes. Depending on the concentration of the molecular target, different design approaches can be used for the synthesis of the molecular probe. If the molecular target is highly expressed and localized in the extracellular space, a simple probe design can be used, e.g. the target specific peptide can be linked to a single gadolinium DTPA. However, if the molecular target is scarce, larger nanoparticles with more than 90,000 gadolinium ions per particle can be used $[19,20]$.

\section{Preclinical and Clinical Molecular MRI Imaging in Cardiovascular Diseases}

\author{
Imaging of Atherosclerotic Plaque Burden \\ and Extracellular Matrix Components
}

Various reports, including some recently published studies $[2 \bullet \bullet, 4]$, have shown that atherosclerotic plaque burden is a specific marker for unstable atherosclerotic plaques. The atherosclerotic plaque burden (Figs. 2,3) is a reflection of overall plaque volume, which is the combined result of the positive and negative remodeling of the atherosclerotic plaque. Conventional luminographic techniques, e.g. X-ray catheter angiographies, only allow the assessment of the degree of stenosis. Hence, the differentiation of stable and unstable atherosclerotic plaques is not possible with this method. It currently remains challenging to determine the atherosclerotic plaque burden in clinical practice. The currently established methods are based on invasive techniques like intravascular ultrasound (IVUS). The high prognostic value of plaque burden for the detection of unstable atherosclerotic plaques has therefore been derived from large-scale invasive studies using intravascular ultrasound techniques $[2 \bullet \bullet, 21]$. It was recently shown that the atherosclerotic plaque burden can also be assessed using molecular MRI [22••]. Using a novel small molecular weight elastin specific MR probe, the atherosclerotic plaque burden can be assessed noninvasively [22••, 23]. This was demonstrated in a mouse model (Fig. 4), as well as in the coronaries of the swine model (Fig. 5). Area measurements derived from noninvasive measurements using this molecular probe correlated well with area measurements derived from ex vivo histological measurements. The focus of these studies was not only to demonstrate the feasibility of quantification of atherosclerotic plaque burden in a preclinical setting, but also to demonstrate the viability of this approach in coronary arteries using a clinical MRI system and clinically applicable MRI sequences. A different molecular MR probe, which allows the visualization of extracellular matrix components, is Gadofluorine [24]. Different components of the atherosclerotic plaque can be identified in an animal model using this molecular probe (Fig. 6), including lipid rich areas [25], neovessel-rich regions [26], and regions rich in fibrous material (collagen) [27].

If plaque rupture occurs, an intraluminal thrombus is formed, which occludes the downstream artery and results in a myocardial infarction or cerebral stroke. Direct visualization of the intraluminal thrombus is feasible with noncontrast enhanced $\mathrm{T} 1$ weighted MR imaging sequences (Fig. 7) [58]. This imaging approach utilizes the high methemoglobin content within the thrombus, which leads to a stronger shortening of the $\mathrm{T} 1$ relaxation time, and thus images of the thrombus as a bright spot.

Fibrin is another promising molecular target in the context of atherosclerotic plaque progression and rupture [27, 28]. Fibrin plays an important role during the initial formation and the subsequent progression of atherosclerotic plaques [22••, 29]. Also, fibrin is a key protein in thrombus formation following the rupture of the fibrous cap of an atherosclerotic plaque. Different molecular probes have been successfully developed to image this protein $[19,30]$. One of the most promising candidates is a small molecularweight fibrin-specific molecular probe, which is comparable to clinically used gadolinium-based contrast agents with respect to size and biokinetic properties $[15,31]$. Due to its high relaxivity, this molecular MR probe has a high sensitivity for the visualization of fibrin within the atherosclerotic plaque and the thrombus. In preclinical studies, coronary in-stent thrombosis was visualized in an experimental swine model [29]. As a result of this successful preclinical evaluation, this small fibrin-specific gadoliniumbased probe was translated into the clinical phase. It was the first molecular MR probe shown to be feasible in preclinical experiments as well as clinical applications [32]. The probe was successfully applied and evaluated in patients for the molecular in vivo detection of cardiac, carotid and aortic fibrin-rich thrombi [32]. Other types of fibrin-specific MR contrast agents, including larger nanoparticles, have also been developed and preclinically tested. Due to their composition, however, most of these agents could not be translated into clinical patient studies [19].

Imaging of Atherosclerotic Inflammatory Burden, Endothelial Markers and Intraplaque

Neovascularization

Inflammatory processes are part of the key biologic events that play an important role in the initiation, development 


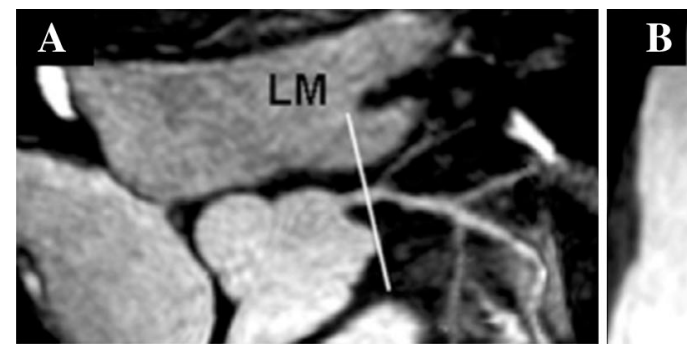

Fig. 2 Assessment of coronary plaque burden and arterial remodeling in patients using cardiovascular magnetic resonance imaging (MRI). In this study, the wall of the coronary arteries was characterized using unenhanced MRI sequences. a Magnetic resonance angiography of the left main coronary artery. b, c Corresponding cross-sections of the arterial lumen and the arterial wall. For
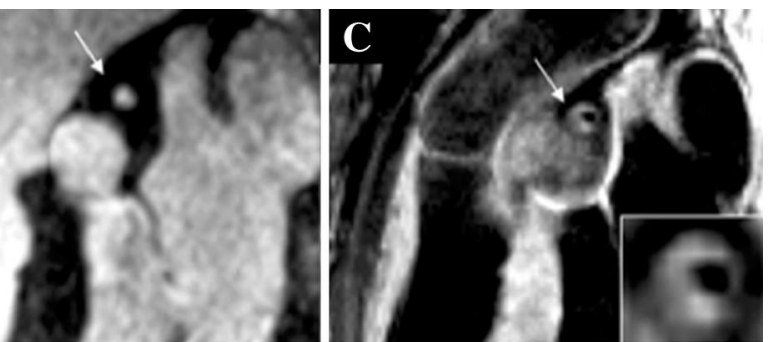

imaging of the coronary vessel wall, a T2 weighted imaging sequence was used. This technique allowed the clear quantification of the positive and negative remodeling of the arterial wall and the assessment of the coronary plaque burden. Adapted from [55], with permission
Fig. 3 Imaging of the coronary artery wall using radial imaging techniques. Magnetic resonance angiography (a) and vessel wall b images of the course of the right coronary artery. In this study, a radial MR imaging technique was used. The right coronary artery including its vessel wall is imaged in its almost complete length from the origin in the aortic wall (Ao) to its distal segments. Adapted from [56], with permission
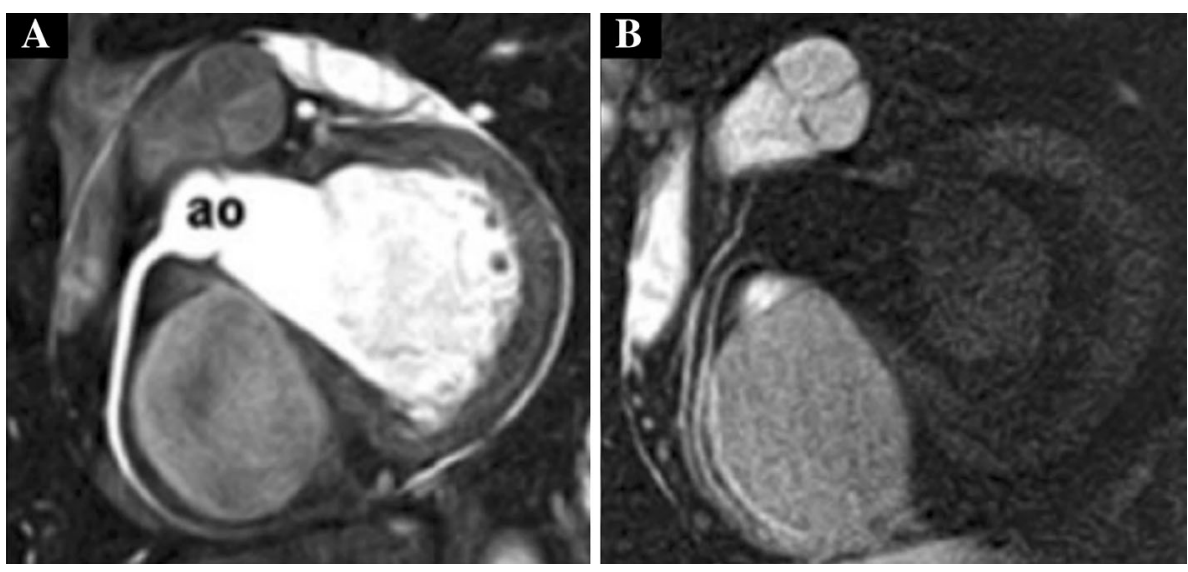
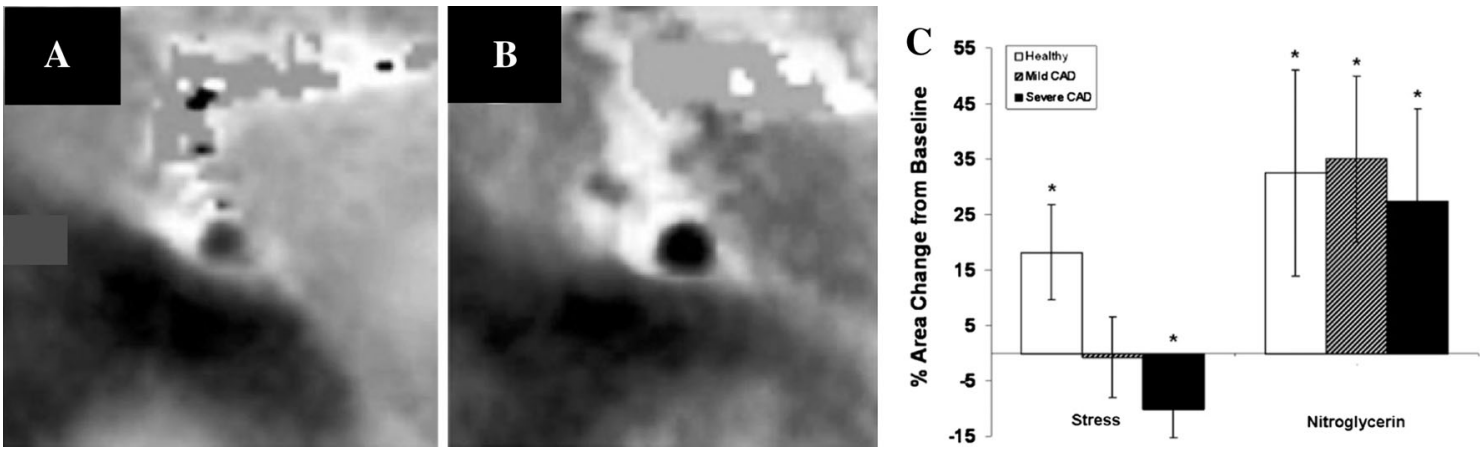

Fig. 4 Molecular imaging of atherosclerotic plaque burden in a preclinical model after administration of an elastin binding probe. a In vivo MR images of the arterial wall in a mouse model of atherosclerosis. On the left, enhancement of the normal arterial wall following administration of the elastin specific contrast agent in a control animal can be appreciated. In the bottom row, fusion of the contrast enhanced images with a time of flight angiography, confirming the localization of the probe in the anterior wall. In the middle row, significant increase in atherosclerotic plaque burden can be appreciated after the administration of the molecular probe. In vivo

and destabilization of atherosclerotic plaques [8•]. Inflammatory cells, e.g. monocytes and leukocytes, from the blood stream migrate into the vascular intima and measurements of plaque burden significantly correlated with ex vivo histological measurements. On the left, images after treatment with anti-inflammatory medication. The molecular probe allowed the clear quantification of the response to treatment. $\mathbf{b}$ Immunoblots confirming the progressive expression of elastin during the development of the atherosclerotic plaque and a reduced expression after treatment. c Chemical structure of the elastin-specific molecular probe. d Electron microscopy confirmed the colocalization of the molecular probe with elastic fibers in the atherosclerotic plaque. Adapted from [22], with permission

differentiate into macrophages. They are the most abundant proinflammatory cells within the matrix of the plaque. One of the key features of vulnerable plaques is a high density 

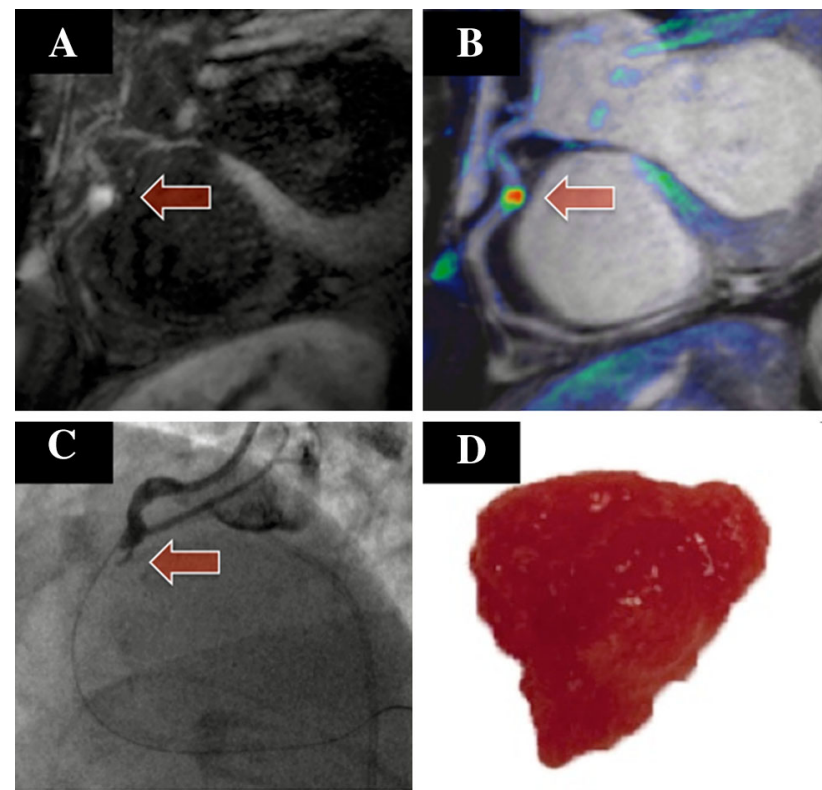

Fig. 5 Assessment of coronary remodeling with molecular MR in a clinical imaging setting. The left image demonstrates a clear enhancement of the remodeled coronary artery wall (LAD) after the administration of the elastin specific molecular probe. All images were acquired in a clinical MRI system using clinically applicable imaging sequences in a large animal model. The molecular probe allowed the quantification of coronary remodeling, which was confirmed by histology. In the middle row, the corresponding coronary MR angiography can be seen. On the left, the contrast enhanced image after the administration of the elastin specific probe. LAD indicates left anterior descending artery; MRA indicates magnetic resonance angiography; $\mathrm{Ph}$ indicates phenylalanine; and RV indicates right ventricle. Adapted from [59•], with permission

of macrophages in the fibrous cap [8•,33]. The detection and quantification of these proinflammatory cells has been shown to be especially useful for the differentiation of stable and unstable atherosclerotic plaques. As discussed earlier in this article, the detection of macrophages using iron-oxide-based probes has become one focus of molecular MRI in the past years [34]. The first studies demonstrating successful in vivo imaging of macrophages in atherosclerotic plaques were published more than a decade ago in an experimental model $[35,36]$. Since then, various studies have investigated and successfully demonstrated the feasibility of the quantification of the inflammatory burden of the atherosclerotic plaque using iron oxide particles $[34,37]$. It was also shown that response to antiinflammatory therapies could be assessed noninvasively using iron oxide particles [37]. One potential drawback of $\mathrm{T} 2$ and $\mathrm{T} 2 *$ shortening probes is that they can only be detected by the resulting signal void in the acquired image. Signal voids can, however, also be caused by tissue interfaces and motion artifacts. Therefore, it can be challenging for the reader to accurately detect these particles in the acquired image. Due to these limitations, novel positive
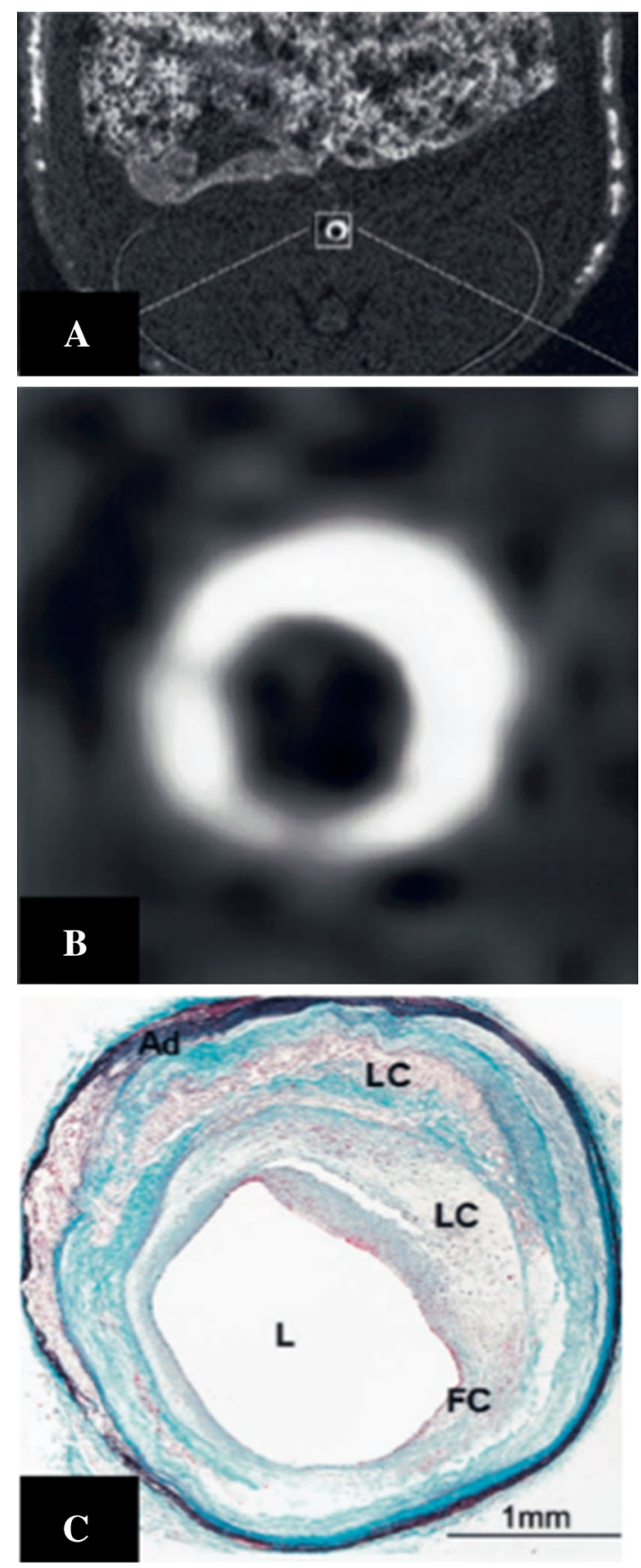

Fig. 6 Molecular imaging of the atherosclerotic plaque in an animal model. In this study, gadofluorine, a gadolinium based molecular MR probe, was used for imaging the distribution of atherosclerosis (a, magnification b) in the aortic wall of a rabbit model. The corresponding histological sections c confirmed the atherosclerotic plaque at that location. $L$ lumen, $A d$ adventitia, $L C$ lipid core, $F C$ fibrous cap. Adapted from [26], with permission

contrast techniques have been developed (IRON, SGM, GRASP). These sequences or postprocessing techniques allow the sensitive detection of iron oxide particles with a positive contrast. They also enable the differentiation of signal voids caused by iron oxide particles from signal voids caused by imaging artifacts [23, 38, 39]. 

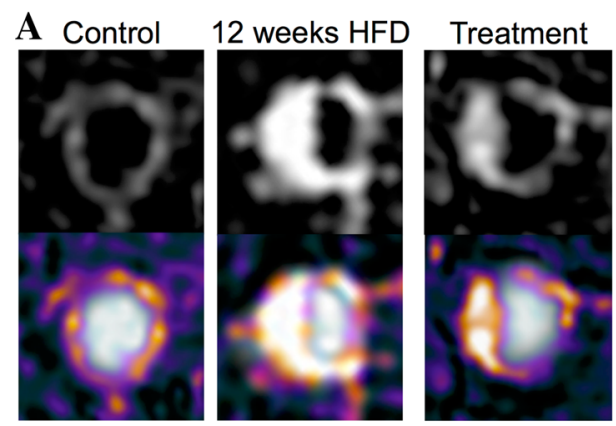

C

B

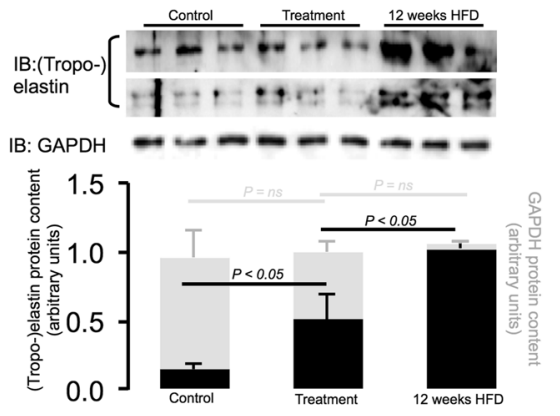

D
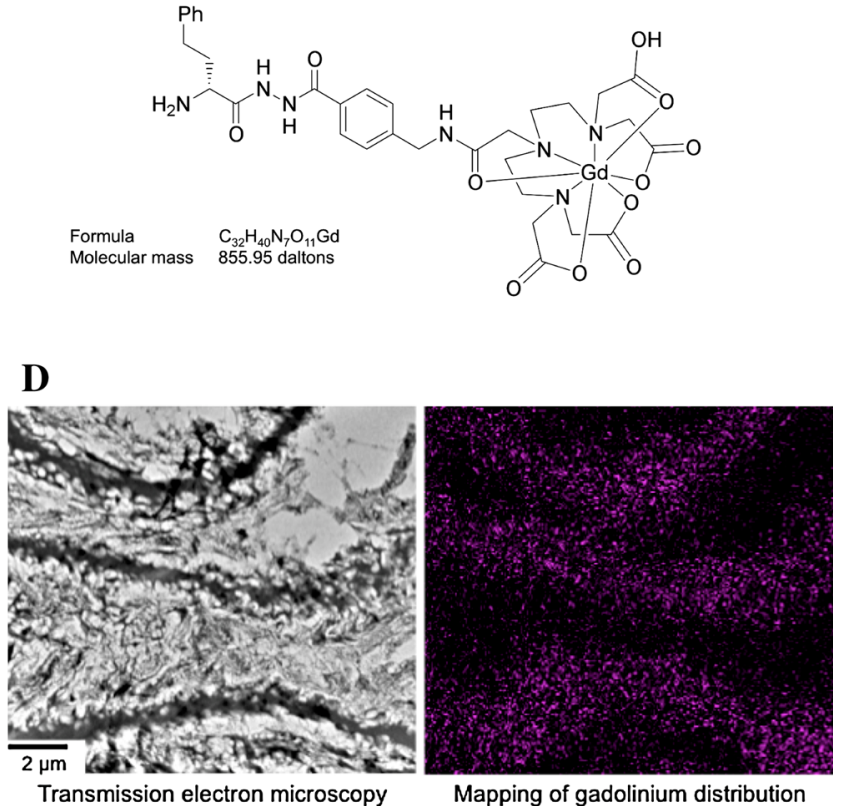

Fig. 7 The direct visualization of intracoronary thrombosis using native magnetic resonance imaging sequences. a The red arrow indicates a thrombus in the right coronary artery of a patient. The thrombus appears bright in the strongly T1 weighted MR image, due to the strong $\mathrm{T} 1$ shortening effect of methemoglobin in the intraluminal thrombus. b Image fusion with magnetic resonance

Adhesion molecules, which are expressed by endothelial cells, are another class of biomarkers used to evaluate the inflammatory state of the atherosclerotic plaque. The most prominent and frequently investigated surface molecules in use are E selectin and the intracellular adhesion molecule 1 (VCAM-1). The main role of these surface molecules is to attract proinflammatory cells from the blood stream. Different molecular MR probes have been developed and extensively studied in different models [40, 41]. The main advantage of this type of molecular target is that it is expressed on the endothelial surface of the plaque, and is therefore directly accessible for intravenously administered molecular probes. Using a VCAM-1 specific molecular probe allows the sensitive detection of these surface markers in atherosclerotic lesions in the aortic arch in $\mathrm{T} 2 *$ weighted sequences [42].

The endothelium's ability to dilate in response to exercise or pharmacological stimuli is an established clinical parameter for the assessment of coronary atherosclerosis. In clinical practice, this parameter is determined with invasive methods (X-ray catheter angiography). Recently, however, it was shown that this parameter can also be assessed noninvasively with MRI [57••]. In this study, a non-contrast enhanced imaging approach was chosen. The authors demonstrated that coronary artery dilation and angiography confirms the intraluminal localization of the thrombus. c Corresponding X-ray angiography, which demonstrates the almost complete luminal stenosis resulting from the thrombus. d Image of the thrombus, which was removed by catheter-based thrombosuction. Adapted from [58], with permission

coronary artery flow assessment with a novel imaging approach is feasible (Fig. 8). The technique allowed the detection and quantification of lacking coronary response to stress patients with coronary atherosclerosis, a clinically established marker of coronary atherosclerosis. Results of this noninvasive non-contrast enhanced imaging approach were comparable to invasive measurements using X-ray angiography $[57 \bullet \bullet]$.

Another very interesting approach for the characterization of atherosclerotic lesions is the detection of intraplaque neovascularization. During the progression of atherosclerotic plaques, local hypoxia triggers the development of new vessels within the matrix of the plaque and the media of the arterial wall. Various studies have shown that the density of these newly formed vessels correlates with the instability of the plaque [43, 44]. An abundantly expressed marker for these newly developed vessels is the unique surface integrin $\alpha_{v} \beta_{3}$. This marker is only expressed on the surface of newly formed endothelial cells. This integrin is not expressed by "regular" vessels in healthy tissues [20]. Two distinct imaging strategies can be used to detect and quantify the process of angioneogenesis [45]. The direct approach is based on specific molecular MR probes, which target the $\alpha_{v} \beta_{3}$ integrin. It was successfully shown that angioneogenesis can be detected in vivo in a 


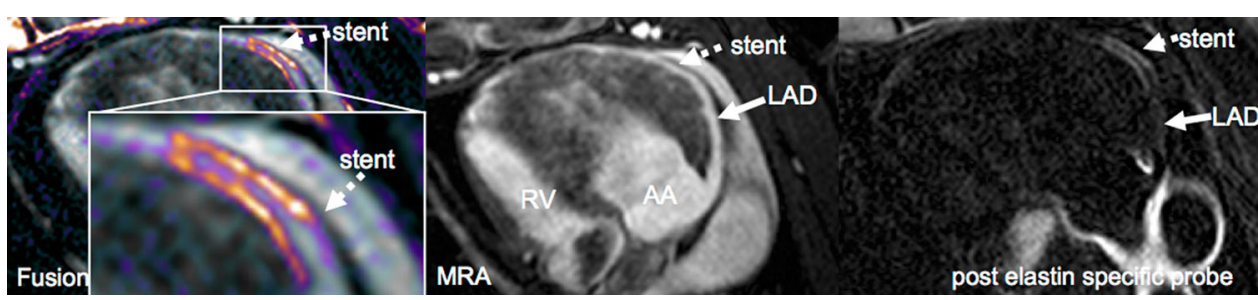

Fig. 8 Noninvasive non-contrast enhanced MRI of coronary artery dilation and coronary artery flow velocity in patients. a Short axis of the right coronary artery of a patient encoded for flow velocity at baseline. b Corresponding image following cardiovascular stress induced by isometric handgrip exercise. $\mathbf{c}$ The charts clearly visualize the differences in coronary blood flow during rest and stress. This study showed that in patients with coronary atherosclerosis, the lack of coronary response to stress could be quantified. This is of high significance, as this is a clinically accepted criterion for the evaluation of coronary atherosclerosis, which can currently only be assessed using invasive methods. Adapted from [57], with permission

long clearance time; in some cases, more than $24 \mathrm{~h}$ [54]. Various groups are working on approaches to overcome these limitations. An alternative and similarly elegant method is the indirect detection of these newly formed vessels by measuring the increase in blood flow after the administration of an unspecific contrast agent [46]. This technique is referred to as dynamic contrast enhanced MRI and can be performed with clinically approved conventional contrast agents. The signal intensity measured in these perfusion scans was shown to correlate with the degree of neoangiogenesis within the plaque. Clinically approved intravascular contrast agents, e.g. the albumin binding gadofosveset trisodium, have been shown to be especially promising for the visualization and quantification of intraplaque angioneogenesis, as well as for the assessment of endothelial permeability [47-49].

Regarding clinical translation of the introduced molecular probes in this chapter, iron oxide particles are the most widely investigated MR probes in patients with atherosclerotic vessel wall changes. In a recent study, it was shown that the signal void, which results from the accumulation of iron oxide particles, is associated with unstable atherosclerotic plaques [50]. A different study has shown the amount of iron oxide accumulation in plaques to closely correlate with the amount of macrophage infiltration [51]. Another significant study demonstrated that patients with symptomatic disease have a higher uptake of iron oxide particles into the plaque compared to asymptomatic patients [52]. An additional demonstrated that response to therapy could be evaluated noninvasively using iron oxide particles [53]. Specifically, it was shown that the uptake of iron oxide particles was significantly reduced following high-dose statin therapy compared to low-dose therapy. These studies indicate the great potential that lies in the application of these molecular probes regarding disease detection, characterization and monitoring of response to therapy. One potential drawback of iron oxide particles that currently limits their wider clinical application is relatively

\section{Summary and Conclusion}

Atherosclerosis is a slowly developing disease, and in some cases it can take decades until individuals become symptomatic. This vast diagnostic window allows the unique opportunity for noninvasive imaging modalities to detect this disease and thereby provide a basis for primary preventive treatment. Currently treatment decisions in the context of atherosclerotic disease are based on the degree of stenosis, which is typically assessed by X-ray catheter angiography. However, increasing evidence is suggesting that the degree of stenosis is not a clinically relevant parameter for the distinction between stable and unstable atherosclerotic plaque. This is of high clinical significance, as most myocardial infarctions and ischemic strokes result from atherosclerotic plaques, which are not associated with a high degree of stenosis. And so, with the luminographic diagnostic techniques and treatment guidelines in use today, these plaques are not detected and therefore not treated.

During the last decade, MRI has made significant progress and has demonstrated high potential for the in vivo characterization of atherosclerotic vessel wall disease. Various studies have shown that MRI allows the detection of early atherosclerotic vessel wall changes, positive vascular remodeling and plaque burden in patients with atherosclerosis. These noninvasive measurements may help to improve the risk stratification of patients and to initiate preventive therapies. Apart from the detection of atherosclerotic vascular changes, MRI also allows the evaluation of response to therapy. All of these parameters can be assessed without the use of contrast agent. And with administration of clinically approved contrast agents, 
further information regarding the relative composition of the atherosclerotic plaque can be gained.

\section{Compliance with Ethics Guidelines}

Conflict of Interest T. C. Walter, G. Knobloch, M. Kasner, G. Diederichs, B. Hamm, U. Ebersberger, and M. R. Makowski declare that they have no conflicts of interest.

Human and Animal Rights and Informed Consent This article does not contain any studies with human or animal subjects performed by the authors.

\section{References}

Papers of particular interest, published recently, have been highlighted as:

- Of importance

- Of major importance

1. Roger VL, Go AS, Lloyd-Jones DM, et al. Heart disease and stroke statistics-2011 update: a report from the American Heart Association. Circulation. 2011;123:e18-209.

2. - Stone GW, Maehara A, Lansky AJ, et al. A prospective natural-history study of coronary atherosclerosis. New Engl J Med. 2011; 364:226-35. This study is of high importance, as it demonstrates that the degree of luminal stenosis is not a valid parameter for the detection of vulnerable plaques. However, other parameters, including plaque burden and the size of the necrotic core, seem to allow such a distinction. In this study, data were assessed using intravascular ultrasound.

3. Ambrose JA, Tannenbaum MA, Alexopoulos D, et al. Angiographic progression of coronary artery disease and the development of myocardial infarction. J Am Coll Cardiol. 1988;12:56-62.

4. Burke AP, Kolodgie FD, Farb A, Weber D, Virmani R. Morphological predictors of arterial remodeling in coronary atherosclerosis. Circulation. 2002;105:297-303.

5. Varnava AM, Mills PG, Davies MJ. Relationship between coronary artery remodeling and plaque vulnerability. Circulation. 2002;105:939-43.

6. Glagov S, Weisenberg E, Zarins CK, Stankunavicius R, Kolettis GJ. Compensatory enlargement of human atherosclerotic coronary arteries. N Engl J Med. 1987;316:1371-5.

7. Rentrop KP. Thrombi in acute coronary syndromes : revisited and revised. Circulation. 2000;101:1619-26.

8. - Libby P, Ridker PM, Hansson GK. Progress and challenges in translating the biology of atherosclerosis. Nature. 2011; 473:317-25. This study is important as it summarizes the key biological features of stable and vulnerable atherosclerotic plaque.

9. Kolodgie FD, Gold HK, Burke AP, et al. Intraplaque hemorrhage and progression of coronary atheroma. New Engl J Med. 2003;349:2316-25.

10. Keenan NG, Locca D, Varghese A, et al. Magnetic resonance of carotid artery ageing in healthy subjects. Atherosclerosis. 2009;205:168-73.

11. Virmani R, Burke AP, Farb A, Kolodgie FD. Pathology of the vulnerable plaque. J Am Coll Cardiol. 2006;47:C13-8.

12. Johnson GA, Benveniste H, Black RD, Hedlund LW, Maronpot RR, Smith BR. Histology by magnetic resonance microscopy. Magn Reson Q. 1993;9:1-30.
13. Choudhury RP, Fuster V, Fayad ZA. Molecular, cellular and functional imaging of atherothrombosis. Nat Rev Drug Discov. 2004;3:913-25.

14. Weissleder R, Elizondo G, Wittenberg J, Rabito CA, Bengele $\mathrm{HH}$, Josephson L. Ultrasmall superparamagnetic iron oxide: characterization of a new class of CONTRAST agents for MR imaging. Radiology. 1990;175:489-93.

15. Caravan P, Ellison JJ, McMurry TJ, Lauffer RB. Gadolinium(III) chelates as MRI contrast agents: structure, dynamics, and applications. Chem Rev. 1999;99:2293-352.

16. Farrar CT, Dai G, Novikov M, et al. Impact of field strength and iron oxide nanoparticle concentration on the linearity and diagnostic accuracy of off-resonance imaging. NMR Biomed. 2008;21:453-63.

17. Bulte JW, Kraitchman DL. Iron oxide MR contrast agents for molecular and cellular imaging. NMR Biomed. 2004;17:484-99.

18. Ferrucci JT, Stark DD. Iron oxide-enhanced MR imaging of the liver and spleen: review of the first 5 years. AJR Am J Roentgenol. 1990;155:943-50.

19. Flacke S, Fischer S, Scott MJ, et al. Novel MRI contrast agent for molecular imaging of fibrin: implications for detecting vulnerable plaques. Circulation. 2001;104:1280-5.

20. Winter PM, Morawski AM, Caruthers SD, et al. Molecular imaging of angiogenesis in early-stage atherosclerosis with alpha(v)beta3-integrin-targeted nanoparticles. Circulation. 2003;108:2270-4.

21. Wu X, Maehara A, Mintz GS, et al. Virtual histology intravascular ultrasound analysis of non-culprit attenuated plaques detected by grayscale intravascular ultrasound in patients with acute coronary syndromes. Am J Cardiol. 2010;105:48-53.

22. •- Makowski MR, Wiethoff AJ, Blume U, et al. Assessment of atherosclerotic plaque burden with an elastin-specific magnetic resonance contrast agent. Nat Med. 2011;17:383-8. This study demonstrated that MRI in combination with a molecular probe allows the reliable noninvasive assessment of atherosclerotic plaque burden, which is a feature of vulnerable atherosclerotic plaques.

23. Makowski MR, Varma G, Wiethoff A, et al. Non-Invasive Assessment of Atherosclerotic Plaque Progression in ApoE-/Mice Using Susceptibility Gradient Mapping. Circ Cardiovasc Imaging. 2011;4:295-303.

24. Meding J, Urich M, Licha K, et al. Magnetic resonance imaging of atherosclerosis by targeting extracellular matrix deposition with Gadofluorine M. Contrast Media Mol Imaging. 2007;2:120-9.

25. Sirol M, Itskovich VV, Mani V, et al. Lipid-rich atherosclerotic plaques detected by gadofluorine-enhanced in vivo magnetic resonance imaging. Circulation. 2004;109:2890-6.

26. Sirol M, Moreno P, Purushothaman K, et al. Increased neovascularization in advanced lipid-rich atherosclerotic lesions detected by gadofluorine-M-enhanced MRI: implications for plaque vulnerability. Circ Cardiovasc Imaging. 2009;2:391-6.

27. Ronald JA, Chen Y, Belisle AJ, et al. Comparison of gadofluorine$\mathrm{M}$ and Gd-DTPA for noninvasive staging of atherosclerotic plaque stability using MRI. Circ Cardiovasc Imaging. 2009;2:226-34.

28. Tavora F, Cresswell N, Li L, Ripple M, Burke A. Immunolocalisation of fibrin in coronary atherosclerosis: implications for necrotic core development. Pathology. 2010;42:15-22.

29. Botnar RM, Buecker A, Wiethoff AJ, et al. In vivo magnetic resonance imaging of coronary thrombosis using a fibrin-binding molecular magnetic resonance contrast agent. Circulation. 2004;110:1463-6.

30. Yu X, Song SK, Chen J, et al. High-resolution MRI characterization of human thrombus using a novel fibrin-targeted paramagnetic nanoparticle contrast agent. Magn Reson Med. 2000;44:867-72.

31. Vymazal J, Spuentrup E, Cardenas-Molina G, et al. Thrombus imaging with fibrin-specific gadolinium-based MR contrast agent 
EP-2104R: results of a phase II clinical study of feasibility. Invest Radiol. 2009;44:697-704.

32. Spuentrup E, Botnar RM, Wiethoff A, et al. MR imaging of thrombi using EP-2104R, a fibrin specific contrast agent: initial results in patients. Eur Radiol. 1911;2008(18):1995-2005.

33. Carr SC, Farb A, Pearce WH, Virmani R, Yao JS. Activated inflammatory cells are associated with plaque rupture in carotid artery stenosis. Surgery. 1997;122:757-63.

34. Kooi ME, Cappendijk VC, Cleutjens KB, et al. Accumulation of ultrasmall superparamagnetic particles of iron oxide in human atherosclerotic plaques can be detected by in vivo magnetic resonance imaging. Circulation. 2003;107:2453-8.

35. Ruehm SG, Corot C, Vogt P, Kolb S, Debatin JF. Magnetic resonance imaging of atherosclerotic plaque with ultrasmall superparamagnetic particles of iron oxide in hyperlipidemic rabbits. Circulation. 2001;103:415-22.

36. Schmitz SA, Coupland SE, Gust R, et al. Superparamagnetic iron oxide-enhanced MRI of atherosclerotic plaques in Watanabe hereditable hyperlipidemic rabbits. Invest Radiol. 2000;35: 460-71.

37. Morishige K, Kacher DF, Libby P, et al. High-resolution magnetic resonance imaging enhanced with superparamagnetic nanoparticles measures macrophage burden in atherosclerosis. Circulation. 2010;122:1707-15.

38. Korosoglou G, Weiss RG, Kedziorek DA, et al. Noninvasive detection of macrophage-rich atherosclerotic plaque in hyperlipidemic rabbits using "positive contrast" magnetic resonance imaging. J Am Coll Cardiol. 2008;52:483-91.

39. Mani V, Briley-Saebo KC, Hyafil F, Fayad ZA. Feasibility of in vivo identification of endogenous ferritin with positive contrast MRI in rabbit carotid crush injury using GRASP. Magn Reson Med. 2006;56:1096-106.

40. McAteer MA, Schneider JE, Ali ZA, et al. Magnetic resonance imaging of endothelial adhesion molecules in mouse atherosclerosis using dual-targeted microparticles of iron oxide. Arterioscler Thromb Vasc Biol. 2008;28:77-83.

41. Mulder WJ, Strijkers GJ, Griffioen AW, et al. A liposomal system for contrast-enhanced magnetic resonance imaging of molecular targets. Bioconjug Chem. 2004;15:799-806.

42. Nahrendorf M, Jaffer F, Kelly K, et al. Noninvasive vascular cell adhesion molecule-1 imaging identifies inflammatory activation of cells in atherosclerosis. Circulation. 2006;114:1504-11.

43. Falk E. Pathogenesis of atherosclerosis. J Am Coll Cardiol. 2006;47:C7-12.

44. Griffioen AW, Molema G. Angiogenesis: potentials for pharmacologic intervention in the treatment of cancer, cardiovascular diseases, and chronic inflammation. Pharmacol Rev. 2000;52:237-68.

45. Aoki S, Aoki K, Ohsawa S, Nakajima H, Kumagai H, Araki T. Dynamic MR imaging of the carotid wall. J Magn Reson Imaging. 1999;9:420-7.

46. Kerwin WS, O'Brien KD, Ferguson MS, Polissar N, Hatsukami TS, Yuan C. Inflammation in carotid atherosclerotic plaque: a dynamic contrast-enhanced MR imaging study. Radiology. 2006;241:459-68.

47. Pedersen SF, Thrysoe SA, Paaske WP, et al. CMR assessment of endothelial damage and angiogenesis in porcine coronary arteries using gadofosveset. J Cardiovasc Magn Reson. 2011;13:10.
48. Lobbes MB, Heeneman S, Passos VL, et al. Gadofosvesetenhanced magnetic resonance imaging of human carotid atherosclerotic plaques: a proof-of-concept study. Invest Radiol. 2010;45:275-81.

49. Phinikaridou A, Andia ME, Protti A, et al. Noninvasive magnetic resonance imaging evaluation of endothelial permeability in murine atherosclerosis using an albumin-binding contrast agent. Circulation. 2012;126:707-19.

50. Kooi ME, Cappendijk VC, Cleutjens KBJM, et al. Accumulation of ultrasmall superparamagnetic particles of iron oxide in human atherosclerotic plaques can be detected by in vivo magnetic resonance imaging. Circulation. 2003;107:2453-8.

51. Trivedi RA, Mallawarachi C, U-King-Im J-M, et al. Identifying inflamed carotid plaques using in vivo USPIO-enhanced MR imaging to label plaque macrophages. Arterioscler Thromb Vasc Biol. 2006;26:1601-6.

52. Howarth SP, Tang TY, Trivedi R, et al. Utility of USPIOenhanced MR imaging to identify inflammation and the fibrous cap: a comparison of symptomatic and asymptomatic individuals. Eur J Radiol. 2009;70:555-60.

53. Tang TY, Howarth SP, Miller SR, et al. The ATHEROMA (Atorvastatin therapy: effects on reduction of macrophage activity) Study. Evaluation using ultrasmall superparamagnetic iron oxide-enhanced magnetic resonance imaging in carotid disease. J Am Coll Cardiol. 2009;53:2039-50.

54. Tang TY, Patterson AJ, Miller SR, et al. Temporal dependence of in vivo USPIO-enhanced MRI signal changes in human carotid atheromatous plaques. Neuroradiology. 2009;51:457-65.

55. Miao C, Chen S, Macedo R, et al. Positive remodeling of the coronary arteries detected by magnetic resonance imaging in an asymptomatic population: MESA (Multi-Ethnic Study of Atherosclerosis). J Am Coll Cardiol. 2009;53:1708-15.

56. Katoh M, Spuentrup E, Buecker A, Manning WJ, Gunther RW, Botnar RM. MR coronary vessel wall imaging: comparison between radial and spiral k-space sampling. J Magn Reson Imaging. 2006;23:757-62.

57. •• Hays AG, Hirsch GA, Kelle S, Gerstenblith G, Weiss RG, Stuber M. Noninvasive visualization of coronary artery endothelial function in healthy subjects and in patients with coronary artery disease. J Am Coll Cardiol 2010;56:1657-65. This important clinical study showed that coronary artery endothelial function, a clinically established and invasively measured parameter, can also be assessed using advanced clinical MRI sequences.

58. Jansen $\mathrm{CH}$, Perera D, Makowski MR, et al. Detection of intracoronary thrombus by magnetic resonance imaging in patients with acute myocardial infarction. Circulation. 2011;124:416-24.

59. - von Bary C, Makowski M, Preissel A, et al. MRI of coronary wall remodeling in a swine model of coronary injury using an elastin-binding contrast agent. Circ Cardiovasc Imaging 2011;4:147-55. In this study, it was demonstrated that a molecular MR probe can be successfully translated from a preclinical small animal model into a large animal model, in combination with clinical MR imaging conditions. 